\title{
Ueber Siedepunkte der Aethan- und Aethylen-
}

\section{Haloïdverbindungen;}

\author{
van A. Sabanejeff. \\ (Eingelaufer den 10. Noramber 1882.)
}

Im Verlafe meiner Intersuchungen über Acetylenderivate*) habe ich zum erstenmal zwei Abkommlinge desselben, das Tribromäliyien und das Acetyiendibromür, in reinem Zustande dargestellt und ibre Siedepunkte von den bis dahin angegebenen auffallend verschieden gefunden. In Folge dieser Correction ergab sich mir eine höchst interessante Gesetzmäfsigkeit in den Siedepunkisdifferenzen der Bromderivale des Acthans und Aethylens, die sich bei weiterem Vergleich auch fär die übrigen Haloidderivate dieser Kohlenwasserstofle als giltig erwies und sich wahrscheinlich auch auf die des Acetylens ausdehnt. In den Arbeiten der verschiedenen Chemiker, die sich mit den Haloidderivaten des Aethans und Aethylens beschätigten, sind wiederholt Versuche gremacht worden. mit Hülfe des vorhandenen Materials eine solche Regelmälsigkeit herauszufinden, um darauf gestützt, die Siedepunkte bis jetzt noch nicht dargestellter Körper im Voraus zu bestimmen.

Schon lange ist bekannt, dafs die Bromverbindungen höher als die Chlorverbindungen und niedriger als die Jodverbindungen sieden und dafs die Siedepunkte der isomeren Körper um so höher liegen, je symmetriseher die Haloidatome dem Kohlenstoff gegenüber im Molecul vertheilt sind. Man war überzeugt, dafs eine constante Differenz in den Siedepunkten existirt; die bisherigen Vergleiche sind jedoch in solcher Weise angestelt worden, dafs sich keineGescizmäfsigkeit ergeben konnte.

*) Diese Anualen $\% \otimes, 12 \%$ 
Manche Chemiker glaubten, dafs bei den analogen Chlor- und Bromverbindungen die Bifferenz die gleiche sei, andere, wie z. B. Anschütz*), verglichen einerseits die gesättigten und andererseits die ungesätigten. Isomeren mit gleich viel Haloïdatomen im Molecu, nabmen hier die gleiche Differenz an und änderten die Siedewunkte in wilkürlicher Weise ab, da die Angaben üher die Siedepunkte mancher Substanzen sehr differiren. Ausfuhrlicher warde die rrage später von Städ el und $D$ e n $z$ el behandelt. Stadel ${ }^{* *}$ ) verglich die gechlorten Aethanderivate unter sich und hestimmte die Siedepunktsernöhung, die der successive Austausch der Wasserstoffatome durch Chloratome zur Folge hatte. $\mathrm{Er}$ hekan jedoch so differirende Zahlen, dafs sich hieraus keine Schiusse ziehen lasser. So z. B. nimm Stadel an, dafs die Umwandung ter Gruppe $\mathrm{CH}_{2} \mathrm{Cl}$ in $\mathrm{CHCl}_{2}$ im Algemeinen aine Siedepunktserhöhung von $30^{\circ}$ zur Folge habe, seine Tabellen zeigen aber, dafs diesdibe zwischen 30 und $46^{\circ}$ schwankt. Man kann bei einem solchen Vergleich auch gar keine constante bifferenz erwarten, da es auf den Siedepunkt entschieden von grofsem Einfurs ist, ob mit dent benachbarten Kohlenstoflaton nur Chlor oder nur Wasserstof verbunden ist. Derael**) vergleicht in ahnlicher Weise die Siedepunkte der Chtorbrom- and der Bromsubstitutionsproducte des Aethans und Aethylens. Nach seiner Zusammenstellung erhöht bei den Chlorbromderivaten jedes eintretende Bromatom den Siedepunkt um 40 ; so lange sich dasselbe an ein schon mit Halogen beladenes Kohlenstoffatom lagert oder lagern kann, anderifalls un $80^{\circ}$, ferner fand er, dafs der Siedepunkt durch Austausch eines Chlor-gegen ein Bromatom nim $25^{*}$ erhöht wird.

*) Berichte der Rentschon ohemisehen Gasollschaft 1:, 2075.

**) Daselbst 746 .

**) Diese Annalen 18, 210 。 
der Aethan- und Aethylen-Haloidverbindungen. 243

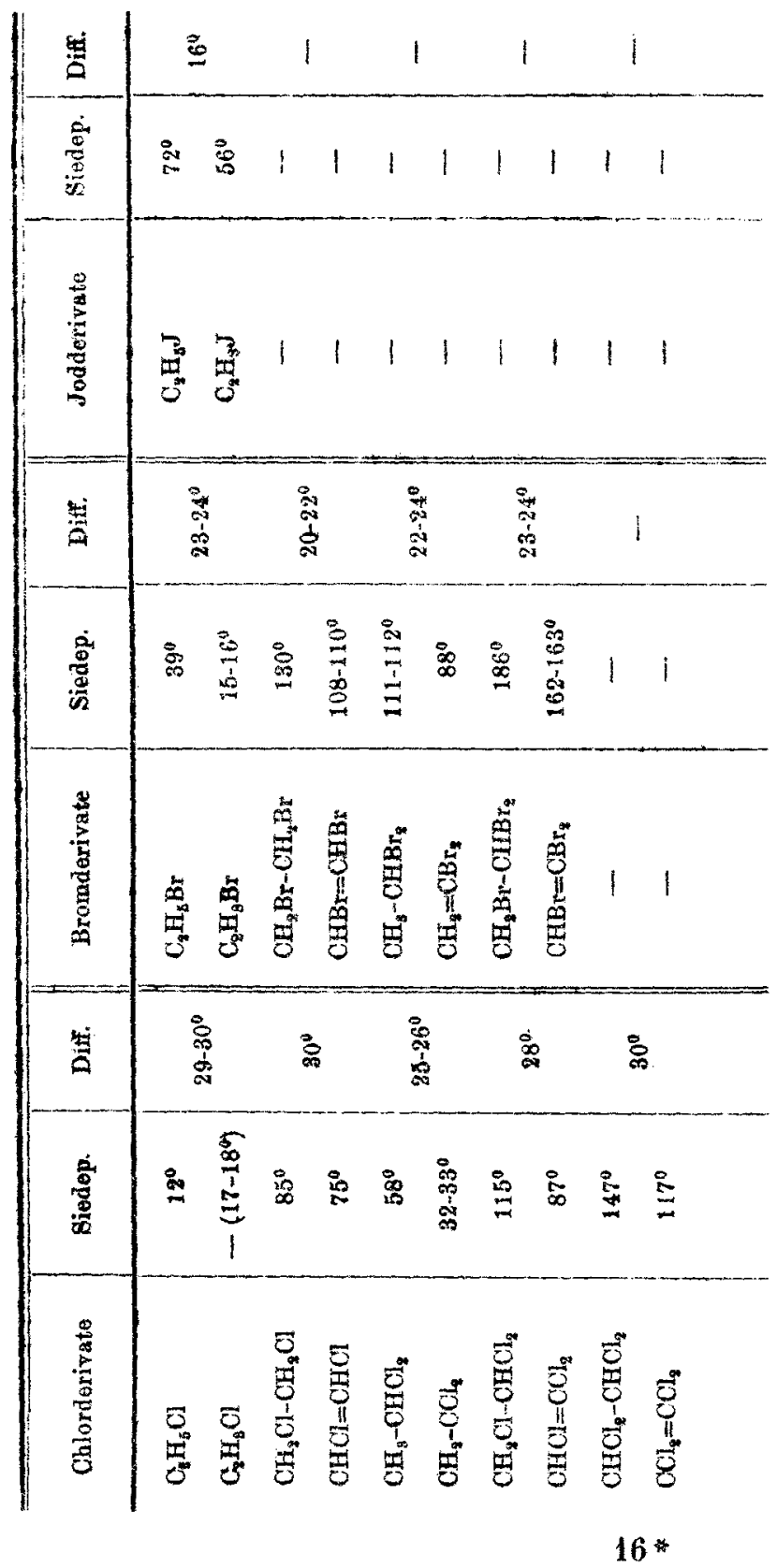


Die oben angedeuteten Siedepunktscorrectionen erlaublen mir in weit einfacherer Weise eine Gesetzmärsigkeit zu constatiren, von der ich glaube, dafs sie einer weiteren Ausdehnung fähig ist.

In vorstehender Tabelle sind die Haloïdderivate des Aethans so zusammengestell, dafs die Substitutionsproducte mit analoger Constitution, d. h. solche, von denen die eine aus der andern durch Verlust $J_{0}$ zwei Wasserstoffatomen entstehen kann, neben einander au stehen kommen (vgl. S. 243) :

\section{Benizrlingen zur Tabelle :}

Chlorderivate : Aethylehlorior siedet nach Regnault (Jahresber. für Chem. u. s. w. f. 1863,70 ) bei $12,5^{\circ}$, wach Linnemann (diese Ann. 106, 195) bei $12,18^{\circ}$ (corr.).

rinylchlorür siedet nach Rega auIt (Jahresber. für Chem. u. s. w. f. 1863,70$)$ bui -17 bis $18^{\circ}$.

Aethylenchlorïr nach l'ierre (Ann. chim. phyo. [3] 20, 5) bei $84,9^{4}$, nach Krämer (Ber. d. d. chem. Ges. 8, 257 u. 261) bei $85^{\circ}$.

Acetylendichlorür nach Bortholot und Jungfleisch (diese Anu. Suppl.-Bd. 7, 252) bei $55^{\circ}$.

Aethylidenchlorür nach Wurtz (Compt. rend. 45, 1013) bei $85^{\circ}$ und nach Bunte (diese Ann. 189, 142) bai $57,5^{\circ}(760 \mathrm{~mm}$ Bar.).

Dichloräthylen nach Regnanlt (diese Ann. 28, 84) bei 35 bis $40^{\circ}$, nach Kramer (Ber. d. d. chem. Ges. 8, 261) bei 370, nach Glinsky's neuen Untersuchungen über Chlorirungspr. des Chlorkthyls und Chloräthylens (Kasan, S. 31, 64, 68) bei 32 bis $33^{\circ}$.

Chloräthylenchlorid nach K r h mer (Ber. d. d. chem. Ges. 8, 257) bei $115^{\circ}$.

Trichloräthylen nach Fischer (Jahresber. für Chem. u. s. w. f. 1864,481 ) hei $87-90^{\circ}$, nach Glinsky (a. a. O.) bei $87^{\circ}$.

Acetylentetrachlorür nach $B$ er thelot und Jungfleisch (a. a. O.) bei 147", nach Paterno und Pisati (Jahresber. für Chem. f. 1871, 507) bei $147^{\circ}$ (corr.).

Dichloräthylchlorür nach Pierre (diese Ann. 80, 125) boi 74,9 nach $S t$ ädel (daselbst 198,180 ) bei $74-75^{\circ}$.

Perchloräthylen nach Regnault (diese Ann. S0, 350) bei 12\%", nach Pierre (Ann. chim. phys. [3] $\mathbf{3}$, 199) bei 123,90, nach 
H. Kolbe (diese Ann. 54, 182) bei $117^{\circ}$ und nach Ge uther (Zoitschr. f. Chem. 1871, 147) bei $116,7^{\circ}$.

Bromderivate: Aethylbromür siedet nack thegnault (Jahresber. für Chom, u. s. พ. f. 1863, 70) boi $38,37^{\circ}$, nach Pierre (Ann. chim. phys. [3] 15, 366) bel $40,7^{\circ}$ u. d. B. $755 \mathrm{~mm}$, nach Linnemann (diese Ann. 169, 195) bei $38,78^{\circ}(760$ mm B.).

Traylbromür siedet nach Robonl (Compt. rand. 74, 947) bei 17 bis $18^{\circ}$, nach Fehling's Handworterbuch 135 bei 15 bis $16^{\circ}(760 \mathrm{~mm} \mathrm{B.})$, nach $\mathrm{Lw}$ ow (Joam. d. russ. chem. Ges. 1878, 236), der die Substanz in grofen Massen darstellke, bei 15 bis $16^{\circ}$.

Aethylenbromïr siodet nach Regnault (dabrobbax, f. Chem. u. s. i. 1863,70$)$ bei $129^{\circ}(752 \mathrm{~mm} \mathrm{B.}$ ).

Acetylendibromitr siedet nach $\mathrm{B} \in \mathrm{rth}$ ol ot hoi $130^{\circ}$. Diese Angabe ist, wie meine Untersuchungen gereigt haben, nurichtig. In meiner ersten Notiz gab ich den Siedep. bei 106 bis $109^{n}$ an, Ansohütz fand thn bei einem nack moiner Methode dargestellten Dibromär boi 110 bis $111^{\circ}$, syuter habe wh die Sabstanz inmer mit dem Siedep. vou 108 bis $110^{\circ}$ bekonmen.

Aethylidenbromür aiedet nach Hofmann (Compt. roxd. 8 $\mathbf{A}, 395$ ) bei $110^{\circ}$, nach Caventou (das $\$$, 1880) bei 110 bis $112^{\circ}$, nacb Reboul (das. 8,947 ) bei $110^{\circ}$, nacb $P a t e r n o$ und Pis ati (Ber. d. d. chem. Ges. 5, 259) boi 110 bis $112^{\circ}$.

Dibromäthylen siedet nach Reboul (diese Arno. 24, 267) bet

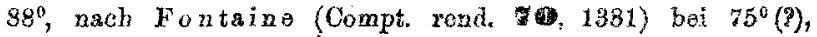
nach Tawildarow (diese Ann. Ta, 28) boi $91 \%$

Bromathylenbromür siedet nach $L \in n n o x$ (diese Ann. 2as, 12a) bai $130^{\circ}$, nach moinen Uniersuchurgen (das. A. 9,128 ) bei 162 bis $163^{\circ}$, nach Demole (Ber. d. d. chom. Ores. H, $318)$ bai 181 bis $183^{\circ}$.

Jodderivate : Aethyljodür nach Linnemann (diese Ana. H 68, 201) bei $72,3^{\circ}$ (corx.).

Vinyljodür nach Regnan It (diese Ann. 63 ) bei $56^{\circ}$.

Wie die Tabelle zeigt sind die Siedepunktedifferenzen der correspondirenden Haloïdderivate des Aethans und Aethylens constant und verändern sich nur mit der Natur. des Halöds, unabhängig von der Menge des letzteren. So differiren die Siedepunkte der Chlorverbindungen um circa $30^{\circ}$, diejenigen des Bromderivate um $23^{\circ}$ und die der Jodverbindungen um $16^{\circ}$. 
Die Siedepunktsdifferenz zwischen Aethyl- und Vinylbromür beträgt 23 bis $24^{\circ}$, zwischen Aethylen- und Acetylenbromür 20 bis $22^{\circ}$, zwischen Aethylidenbromür und Dibromäthylen 22 bis $24^{\circ}$ und endich zwischen Bromäthylenbromür und Tribromäthylen 23 bis $24^{9}$. Man sieht, dafs diese Zahlen sehr wenig von dem Mittelwerth $23^{\circ}$ abweichen.

Beim Vergleich der Siedepunktsdifferenzen der Chlorderivate fält in die Augen, dafs die zwischen Aethylidenchlorür und Dichloräthylen um 4 bis $5^{0}$ kleiner ist als die der übrigen Derivate. Dies erklärt sich aber leichi damit, dafs der Siedepunkt des Dichloräthylens überhaupt nicht genau bekannt ist und in Wirklichkeit um einige Grade tiefer angenommen werden mufs, wie jeder, der die Literaturangaben hierüber genauer surchgeht, zugeben wird.

Von den Jodsubstitutionsderivaten war es vis jetzt nur möglich šrne Differenz anzugeben.

Die Siedepunkte der Haloidderivate des Acetylens, nämlich des Chlor- und Bromacetylens, sind noch nicht bekannt und lassen sich deshalb auch mit denen der entsprechenden Aethylenderivate nicht vergleichen. Indessen sind einige annäherndo Angaben über Bromacetylen vorhanden. Kob oul *), der es mit Acetylen gemischt erhielt, beschreiht es als ein Gas, das sich unter einem Druck ron urei Atmosphären verdichtet.

Nimnt man an, daf. die Siedepunktsdifferenz zwischen inm und Finylbromür die gleiche ist, wie zwischen dem letzteren und dem Aethylbromür, so wird sein Siedepunkt zwischen -7 und -8 fallen. Es läfst sich mit ziemlicher Wahrscheinlichkeit annehmen, dafs auch xwischen den Aethylenand Acelylenderivaten die Siedepunktsdifierenz von $23^{\circ}$ für

*) Diest Annulen 25 , 81 . 
die Brom- und $30^{\circ}$ für die Chlorsubstitutionsproducte vorhanden ist.

Auf Grund dieser Yoraussetzung wird das Chloracetylen, $\mathrm{C}_{2} \mathrm{HCl}$, von -47 bis $-48^{\circ}$, Jodacetylen, $\mathrm{C}_{2} \mathrm{HJ}$, bei $40^{\circ}$, Perchloracetylen, $\mathrm{C}_{4} \mathrm{Cl}_{2}$, bei $25^{\circ}$, Perbromacetylen, $\mathrm{C}_{2} \mathrm{Br}_{2}$, bei 85 bis $87^{\circ}$ sieden; die Siedepunkte von Acetylentetrabromür und Perbromäthylen müssen um $23^{\circ}$ auseinander liegen u. s. w.

Werden die gemischten Haloidderivate des Aethans und Aethylens in ähnlicher Weise in einer Tabelle zusammengestellt, so ergiebt sich folgendes Resultat :

\begin{tabular}{|c|c|c|}
\hline Formel & Siedep. & Differenz \\
\hline $\mathrm{CH}_{\mathrm{g}}-\mathrm{CHBrCl}$ & $85^{\circ}$ & \multirow{2}{*}{$23^{\circ}$} \\
\hline $\mathrm{CH}_{2}=\mathrm{CBrCl}$ & $62^{\circ}$ & \\
\hline $\mathrm{CH}_{2} \mathrm{Br}-\mathrm{CHBrCl}$ & $163^{\circ}$ & \multirow{2}{*}{$21-22^{\circ}$} \\
\hline $\mathrm{CHBr}=\mathrm{CBrCl}$ & $141-142^{\circ}$ & \\
\hline $\mathrm{CH}_{2} \mathrm{Cl}-\mathrm{CH}_{8} \mathrm{Br}$ & $106^{\circ}$ & \multirow{2}{*}{$23-26^{\circ}$} \\
\hline $\mathrm{CHCl}=\mathrm{CHBr}$ & $80-83^{\circ}$ & \\
\hline $\mathrm{CH}_{2} \mathrm{Cl}-\mathrm{CH}_{4} \mathrm{~J}$ & $138^{\circ}$ & \multirow{2}{*}{$23^{0}$} \\
\hline $\mathrm{CHCl}=\mathrm{CHJ}$ & $115^{\circ}$ & \\
\hline $\mathrm{CH}_{3} \mathrm{Br}-\mathrm{CH}_{\mathbf{2}} \mathrm{J}$ & $132^{\circ}$ & \multirow{2}{*}{$17-22^{0}$} \\
\hline $\mathrm{CHBI}=\mathrm{CHJ}$ & $140-145^{\circ}$ & \\
\hline
\end{tabular}

Bernerkungen zur Tabelle :

a-Chlorbromäthan siedet nach Lescoeur (Bull. sos. chim. Paris [2] 20, 483) bei $84,5^{\circ}$.

$\beta$-Chlordibromäthan $\left(\mathrm{CH}_{2} \mathrm{Br}-\mathrm{CHBrCl}\right), \beta$-Chtordibromäthylen (CHBr $=\mathrm{CBrCl})$ und $\alpha$-Chlorbromathylen $\left(\mathrm{CH}_{\mathrm{g}}=\mathrm{CBrCl}\right)$ nach den $\mathrm{An}$ gabon Donzel's (diese Ann. ISE, 210).

B-Chlorbromäthan (Chlorbromäthylen) $\left(\mathrm{CH}_{2} \mathrm{Br}-\mathrm{CH}_{2} \mathrm{Br}\right)$ nach $\mathrm{Simpson}$ (Lond. Roy. Soc. Proc. 2\%, 118 und Jahresber. für Chem. u. s. w. f. 1878, 408) bei 108-110 , nach Lescoour (a. a O.) bei $106^{\circ}$. Die Siedepunkte von $\beta$-Chlorkromathylen (Acetylenchlorabromilor) $(\mathrm{CHCl}=\mathrm{CHBr})$, von $\beta$-Ohlorjodätizlen ( $\mathrm{CHCl}=$ CHJ) (Acetylenchiorojodïr) und ron R-Bromjodäthylen (Acetylenbromojodrir) sind von mir bestimant worden. 
$\beta$-Bromjodithan (Aethylenbromojodïr) siedet nach $\mathbf{R e b o u l ~ ( d i e s e ~}$ Ann. 55, 29 und 212) bei $162^{\circ}$. Die Angaben über den Siedepunkt des $\beta$-Chlorjodäthans sind sehr verschieden. Nach Butlerow und Ossokin (d. Ann. 145, 270) siedet es bei 138 bis $140^{\circ}$, nach Ma um né (Compt. rend. AB, 727) bei $146^{\circ}$, nach Meyer und Wurster (Ber d. d. ehem. Ges. 964) bei 137 bis $138^{\circ}$; die von mir unter den gleichen Bodingungen wie das Acetylenchlorojodür dargestellte Substanz siedete bei 138 bis $139^{\circ}$.

Aus der Tabelle ergiebt sich ungefähr die Siedepunktsdifferenz von $23^{\circ}$. Man könnte hieraus den Schlufs ziehen, dafs dieselbe bei gemischten Haloidderivaten von der Natur des Haloïds mit dem gröfsten Atomgewicht abhängig sei, während sie von den anderen Haloïden nicht beeinflufst wird; hiermit steht jedoch in Widerspruch, dafs die Siedepunktsdifferenz zwischen Aethylenchlorjodïr und Acetylenchlorjodür auch $23^{\circ}$ und nicht $16^{\circ}$ beträgt.

Wie oben erwähnt giebt Denzel an, dafs der Siedepunkt bei den Haloidderivaten des Aethans und Aethylens um 250 durch den Austausch eines Chloratoms gegen ein Bromatom steigt. Eine solche constanle Differenz existirt jedoch nicht, sonst müfsten die Siedepunktsdifferenzen zwischen den Chlorderivaten des Aethans und Aethylens einerseits und die zwischen den Bromderivaten andererseits gleich sein; ich habe aber gezeigt, dafs sie für die ersteren höler $\left(30^{\prime \prime}\right)$ als für die letzeren $\left(23^{\cup}\right)$ sind. In der That schwanken auch die von De nz el angegebenen Siedepunktsdifferenzen in Grenzen von $7^{\circ}$.

Die Verhältnisse, welche die Siedepunkte einer Gruppe von Körpern beeinllussen, können nur dann als vollständig aufgeklärt angenominen werden, wenn es gelingt, von einem Siedepunkte ausgehend den aller anderen Körper dieser Klasse zu bestimmen. Ich habe hier nur den Zusammenhang zwiscben den Siedepunkten der gesättigten und ungesättigten Halöilverbindungen gezeigl. Um die Frage vollständig zu beantworten, wäre es noch nothwendig 1) die Abhängigkeit der Sied`- 
temperatur von der Constitution und 2) die Veränderung derselben durch den Eintritt oder gegenseitigen Austausch der Haloïdatome aufzuklären. Versuche in dieser Richtung sind

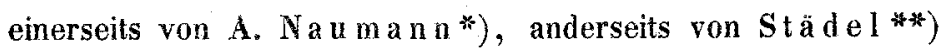
gemacht worden. Die Verhältnisse sind hier sehr complicirt, aber ich glaube, dafs die von mir gefundene Gesetzmärsigkeit uns auch hier einen Schritt vorwärts bringt.

Es war zu erwarten, dafs eine ähnliche Gesetzmäfsigkeit auch bei anderen complicirteren Haloidderivaten vorhanden ist. Vergleicht man in ähnlicher Weise, wie es oben geschehen ist, die Haloidderivate der Kohlenwasserstoffe mit drei Kohlenstoffatomen im Molecul, so zeigen sich bedeutende Schwierigkeiten, sowohl wegen de vorhandenen grofsen lücken und der gröfseren Anzah yon Isomeren, als auch weil einer gesättigten Verbindung mohrere ungesătigte entsprechen können. Siehe die Tabelle S. 250.

Aus der Tabelle ergiebt sich folgendes Resultat : "Treten aus einen Grenthalölderivate, dessen Molecul drei Kohlenstoffatome enthäl, wives Wserstoffatome, welche an halouldreie Kohlenstoffatome gebinden sind, aus, so erleidet die Siedetemperatur fast gor heine Aenderang; in entgegengesetzten Falle, wenn die neben Balouten an einem Kohlensioffatom sitzenden Wasserstoffatome aus dem Molecul entfont werden, wird der Siedepuntit erriedrigt, aber weniger als es bei den Haloüdderivaten des Aethans der Fall ist. So sieden 2. B. die Propylderivale hei derselben Temperatar wie die Allylderivate, whirend die Siedepunkte der Pseudopropylderivate höher als die der correspondirenden Propylenderivate liegen. Im Algemeinen ist die Siedepunhtsdifferenz bei den Chlor-

*) Ber. d. dentsch. chem. Gas. a, 173 u. 206.

**) Daselbst 176. 


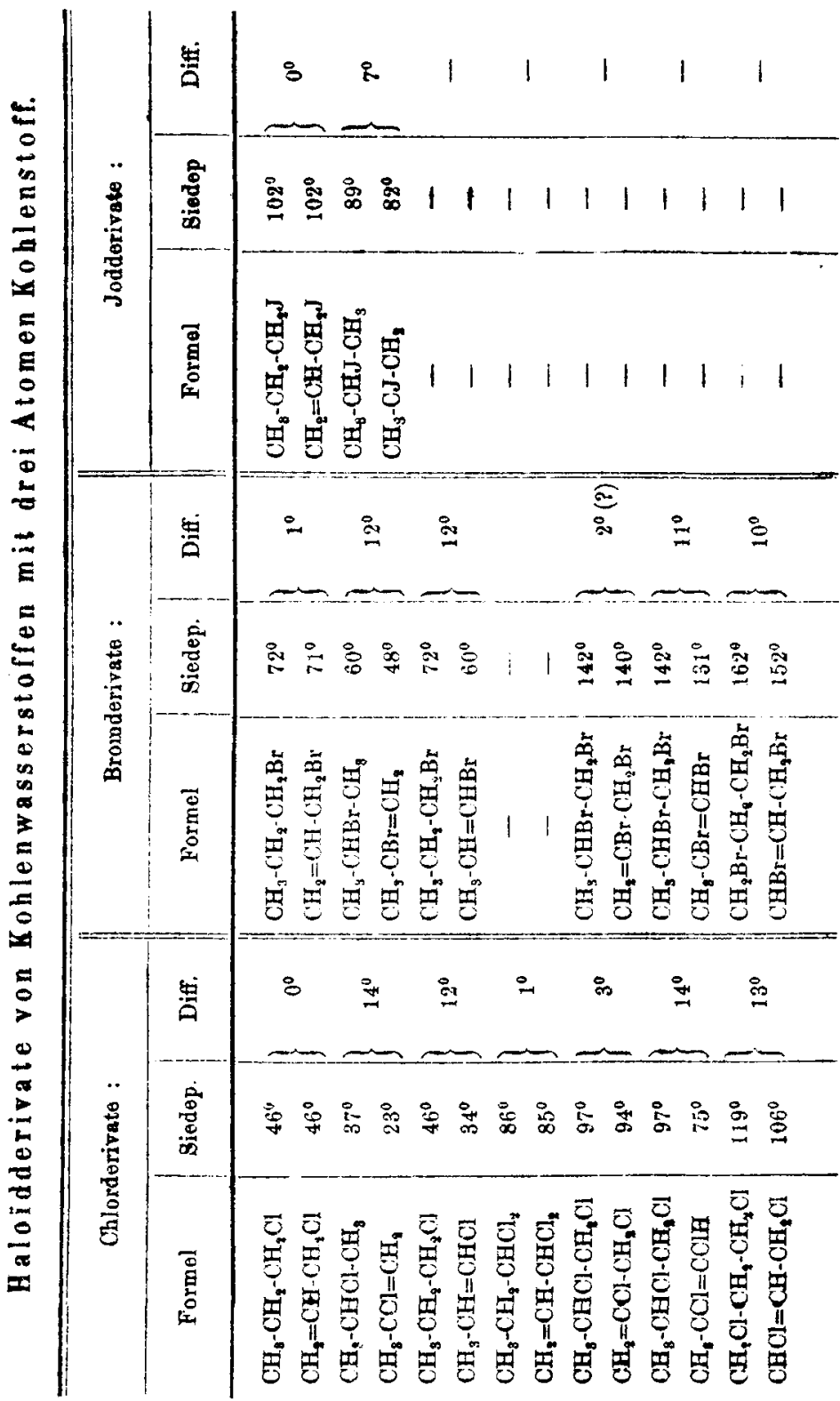


derivaten grölser als bei den Bromderivaten und diese wieder gröfser als bei den Jodderivaten.

Wahrscheinlich findet sich eine ühnliche Regelmärsigkeit auch zwischen den Siedepunktev der gesättigten und ungesuttigten hologenfreien Verbindungen, wie aluch bei den Gliedern isologer Reihen.

\section{$\longrightarrow$ \\ Ueber Acetylenderivate; von Denselben.}

\section{Ueber Darstellnng und Eigensebaften von Acetylen- dibromür.}

In meiner Abhandlung in diesen Annalen 178, 109 sind bereits Versuche beschrieben worden, welche die Darstellung des Acetylendibromürs bezweckten. Als Hauptresultat ergab sich damals, dafs die Bildung desselben vorzüglich in der Anwendung verdünnter Bromlösungen und dem steten Vorhandensein von überschüssigem Acetylen beruht. Indefs verlangt die dort angegebene Darstellungsmethode viel Zeit und die Ausbeute an Dibromür ist äufserst gering, da viel Acetylen unabsorbirt durch den Alkohol hindurchgeht; zudem orwies sich das so erhaltene Product, trotz der ziemlich gut stimmenden Analysen, nicht als vollkommen rein.

Nach mehreren verunglückten Versuchen reines Dibromür in anderer Weise darzustellen, gelang es mir bereits vor mehreren Jahren, dasselle nach dem Vorschlag des Hrn. Prof. Erlenmeyer in dessen Laboratorium auf folgende Weise zu erhalten und seine Eigenschaften näher zu untersuchen. Die Methode beruht in der Einwirkung von Zink und Alkohol 